\title{
Referential processing: Reciprocity and correlates of naming and imaging
}

\author{
ALLAN PAIVIO, JAMES M. CLARK, NANCY DIGDON, and TRUDY BONS \\ University of Western Ontario, London, Ontario, Canada
}

\begin{abstract}
To shed light on the referential processes that underlie mental translation between representations of objects and words, we studied the reciprocity and determinants of naming and imaging reaction times (RT). Ninety-six subjects pressed a key when they had covertly named 248 pictures or imaged to their names. Mean naming and imagery RTs for each item were correlated with one another, and with properties of names, images, and their interconnections suggested by prior research and dual coding theory. Imagery RTs correlated $.56(d f=246)$ with manual naming RTs and .58 with voicekey naming RTs from prior studies. A factor analysis of the RTs and of 31 item characteristics revealed 7 dimensions. Imagery and naming RTs loaded on a common referential factor that included variables related to both directions of processing (e.g., missing names and missing images). Naming RTs also loaded on a nonverbal-to-verbal factor that included such variables as number of different names, whereas imagery RTs loaded on a verbalto-nonverbal factor that included such variables as rated consistency of imagery. The other factors were verbal familiarity, verbal complexity, nonverbal familiarity, and nonverbal complexity. The findings confirm the reciprocity of imaging and naming, and their relation to constructs associated with distinct phases of referential processing.
\end{abstract}

Mental translation between verbal and nonverbal information is an important associative activity. Such code switching has been termed referential processing (Paivio, 1971, 1986) or referential activity (Bucci, 1984; Bucci \& Freeman, 1978), and it is reflected most directly in picture-naming and word-imaging tasks. Referential processing is implicated as well in memory of pictures and words, symbolic comparisons, language comprehension, cognitive deficits after brain damage (e.g., Kosslyn, 1987; Thompson, Hall, \& Sison, 1986), and other cognitive phenomena, and it raises important conceptual issues on which contemporary theories of mental representation differ. Dual coding theory (Paivio, 1971, 1986) maintains that verbal and nonverbal representations are directly connected in a one-to-many fashion in both directions, whereas common coding models assume that crossmodal processing requires activation of shared abstract representations (e.g., Potter \& Faulconer, 1975; Snodgrass, 1984). Research on naming and imaging also addresses specific theoretical issues, as, for example, the question of whether images are constructed sequentially from components (e.g., Kosslyn, 1980) or are activated holistically (e.g., Paivio, 1971, p. 58; 1986, p. 60).

Despite the familiarity and theoretical importance of referential processing, we lack systematic information

This research was supported by grant A0087 to Allan Paivio from the Natural Sciences and Engineering Research Council of Canada. We thank Carla Johnson for helpful comments on earlier drafts of the paper, and Kal Csapo, who collected some of the older data that we used. Ad dress correspondence to Allan Paivio, Department of Psychology, Faculty of Social Sciences, University of Western Ontario, London, Ontario N6A 5C2, Canada. about the relation between the two directions of processing and about the mutual determinants of naming and imaging. The two tasks have been investigated separately in numerous studies (reviewed below) but not together. Similarly, most of the relevant cognitive theories have dealt separately with the two phenomena. The present study provides unique information about the relation between naming and imaging, and about their mutual correlation with predictors suggested by theory and prior research. Specifically, we measured the latency to image to words and to name pictures for a large number of items, and correlated the mean reaction times (RTs) with one another and with 31 item attributes. We expected that correlations over items would reveal both similarities and differences in the two directions of processing and would extend our understanding of the referential mechanisms involved in naming pictures and imaging to words.

Our research was guided by dual coding theory, which includes specific assumptions concerning both directions of referential processing. The theory develops the view that referentially related verbal and nonverbal (i.e., imaginal) representations are directly connected, and that referential processing across the interconnections is probabilistically determined by the number, kind, and recency of one's prior experiences of referent objects and their names (Paivio, 1971, pp. 74-75; 1986, chap. 4), as well as by such contextual variables as task instructions. Conscious verbal and imagery experiences are distinguished conceptually from the underlying cognitive representations for words and of objects, which have accordingly been called logogens (borrowed from Morton, 1969, 1979) and imagens (Paivio, 1986), respectively. Although modality-specific representations are assumed 
to exist within the verbal and imagery systems (Paivio, 1971, pp. 55-56; 1986, chap. 4), the focus of the present paper is on referential connections between visual object codes and auditory-articulatory name codes.

The general assumptions of dual coding and related theories suggest three operational phases to naming and imaging. The first phase of naming is picture identification, in which a nonverbal representation is activated (cf. the Höffding step). That representation in turn activates connections to various verbal representations during a second, referential processing phase. A third phase involves processes by which one of the competing verbal representations produces a response. Imaging occurs in a similar manner, but the stimulus-response roles of the verbal and nonverbal codes are reversed, as is the direction of referential connections. A word first activates a corresponding verbal code through perceptual processing, the verbal code in turn activates referential connections to alternative imagery codes, and an imaginal code corresponding to a specific object is subsequently selected by the convergent influence of relevant variables.

The hypothesis of multiple phases to naming and imaging has been supported empirically. Picture naming is slower than responding in tasks that do not entail referential translation, such as the reading of words (e.g., see Cattell, 1886; Moore, 1915; Potter \& Faulconer, 1975) or the identification of objects (e.g., see Fraisse, 1968; Moore, 1915). Practice reduces but does not erase the reading advantage (Brown, 1915; Fraisse, 1960, 1968), suggesting that structural constraints, such as the hypothesized referential connections, underlie the difference. Similar evidence for multiple phases to imagery has been obtained from image generation tasks (e.g., by Paivio, 1971). Moore (1915), for example, found that imaging RTs were longer than RTs in a control task that required only verbal processing of words.

Other models of naming and imaging differ from dual coding theory in important ways, especially in the greater abstractness of their representational units and the absence of specific assumptions concerning the reciprocity of naming and imaging. Nonetheless, theoretical components and processing phases analogous to referentially connected verbal and nonverbal codes can be identified in multiplestage models of naming (e.g., see Lachman \& Lachman, 1980 ) or imaging. For example, the naming model of Seymour (1973) includes logogen and pictorial access-exit nodes that are interconnected, albeit indirectly, by abstract codes. The Kosslyn (1980, pp. 150, 152) model of imagery likewise uses name codes as inputs to an IMAGE procedure that finds IMG files containing image data, although the model does assume that name codes are abstract propositions and that image data are stored in a nonanalogue form. Some implications of the present results for the abstractness of mental representations are considered in the discussion. Part 1 of this paper deals with the reciprocal relation between referential processing in the verbal-to-nonverbal and nonverbal-to-verbal directions, and Part 2 with specific predictor variables that are assumed to affect naming and imaging during theoretically distinct phases of referential processing.

\section{PART 1: CORRELATION BETWEEN IMAGING AND NAMING REACTION TIMES}

Little is known about the degree of reciprocity between naming and imaging, but theoretical considerations suggest that the ease of the two operations should be positively correlated. This expectation follows from the dual coding assumption that experience plays a major role in the development and maintenance of referential connections, with experiences that strengthen connections in one direction also providing opportunities for strengthening connections in the opposite direction. For example, naming an object would directly strengthen the nonverbal-toverbal processing sequence, but the contingencies could also strengthen verbal-to-imaginal processing, provided that the object or its image persisted after activation of the name. Drawing pictures or imaging would similarly strengthen referential processing in both directions if subjects named during the task. Such reciprocal reinforcing experiences would lead to a correlation between imaging and naming RTs.

Dependencies between naming and imaging RTs also would be expected if the same image and name representations were joined by referential connections in the two directions. Under those conditions, the ease with which a specific imaginal representation is activated, by whatever means, could contribute both to object identification in a naming task and to image production in an imaging task. Name accessibility similarly could facilitate perceptual identification of stimulus words in an imaging task and verbal response production in picture naming. This hypothesis is consistent with evidence that word frequency affects both picture naming and word identification (Wingfield, 1968). However, failure to find transfer from picture naming to word identification tasks (Morton, 1979) suggests that the specific verbal codes used in picture naming may differ from those involved in identifying (and presumably imaging to) visually presented words.

These hypotheses suggest that imaging and naming RTs will be correlated, but their relation is unlikely to be perfect. To the extent that individual referential connections are unidirectional rather than bidirectional, connections in one direction could be differentially developed and strengthened by specific experiences that would not necessarily affect the reverse pathways to the same degree. An imperfect correlation would also be expected because properties of the verbal and nonverbal representations might have asymmetrical effects on stimulus and response processing. For example, despite large effects on picture naming, word frequency sometimes has no effect on reading latency for words (Scarborough, Cortese, \& Scarborough, 1977, Experiment 3), an initial stage in imaging.

The few studies that bear on the hypothesized relation between naming and imaging have investigated variation 
among subjects rather than items and have inferred imagery from indicators that are operationally indirect. Individual differences in referential activity have been measured in terms of differences between the time to name colors and read color words (e.g., by Broverman, 1960; Bucci, 1984; Lazarus, Baker, Broverman, \& Mayer, 1956). According to Bucci, large differences indicate difficulties in translation from nonverbal to verbal codes, and should be associated with poor referential abilities in both directions. Consistent with this hypothesis, referential ability correlates positively with the concreteness of descriptions given for color names and personal experiences (Bucci, 1984), suggesting greater use of imagery by subjects with high referential ability. Reciprocity is also suggested by Thompson et al.'s (1986) finding that imagery practice facilitated subsequent naming by aphasic subjects.

Such results are consistent with the hypothesis that imaging and naming are related operations, but they do not provide very precise information on the degree of reciprocity. Accordingly, we examined the strength of the relation using operationally direct measures of naming and imaging. Subjects mentally named pictures or imaged to words before manually indicating completion of the specified task, and then writing the name or drawing the image. Mean imaging and naming RTs for each item were correlated with one another and with voicekey naming RTs from earlier studies. The voicekey and manual naming RTs provided evidence about convergent validity, and also about the effect of response mode on the expected positive correlation between naming and imaging.

\section{Method}

Subjects. Introductory psychology students, 48 males and $48 \mathrm{fe}$ males, participated to fulfill a course requirement. Nine additional subjects failed to attend Session 2 or experienced mechanical or other problems.

Materials. The stimuli consisted originally of 255 black-and-white line drawings and their names (Csapo, 1971; Paivio, 1973). Many of the pictures were obtained from primary school readers, and most represented familiar objects with common labels for which word frequency values were available in Thorndike and Lorge (1944). Slides were made of white text or line drawings on black backgrounds. All the pictures occupied roughly the same area, and the words appeared in uppercase letters. We subsequently excluded 7 items of a sensitive nature, leaving 248 pictures and words.

Procedure. The subjects participated in two 1-h sessions approximately 1 week apart. Twenty-four subjects were assigned to each of four conditions: naming in both sessions (NN group), imaging in both sessions (II), naming in Session 1 and imaging in Session 2 (NI), or imaging in Session 1 and naming in Session 2 (IN). Two random orders of items were used, with approximately equal numbers of slides in each of four trays. Pictures and corresponding labeis occupied the same positions in their respective trays. For each subject, the order of trays was randomized separately for each session, but the order of items in trays was constant.

In each session, the first 24 subjects ( 12 IN, 12 NI) saw 255 words or pictures projected individually on a screen approximately $3 \mathrm{~m}$ in front of them. One item order was used for these subjects. A timer was activated when each slide was shown; it was stopped when the subject pressed a response key. An experimenter recorded the time and advanced the projector for the next trial. For the remain- ing 72 subjects ( $12 \mathrm{IN}, 12 \mathrm{NI}, 24 \mathrm{NN}, 24 \mathrm{II}), 248$ words or pictures were projected on a white surface $1 \mathrm{~m}$ in front of the subject. Twelve of the subjects in the NN and II conditions saw items in the same order as the earlier IN and NI subjects did. The remaining 48 subjects, 12 in each group, saw the items in the second order When the subjects pressed a key, a computer opened a shutter and started a millisecond timer that stopped when the key was released The computer stored the RTs and advanced the slides. Differences in equipment had little effect on the correlations between imaging and naming RTs (see Results). For all the subjects, the slides remained visible until the subject responded, brief rests occurred during slide tray changes, and each session began with four practice trials.

The picture-naming instructions informed the subjects that they would see a number of pictures one at a time and that they should press (or release) the key as soon as they thought of a name, which they then wrote on a response sheet. The instructions emphasized that it was important to respond quickly, but not until the name of the picture was known. The subjects in the imaging task were instructed to think of an image for each word, to press (or release) the key as soon as they had an image, and then to sketch their image. They were told not to worry about the quality of their drawings.

Voicekey naming RT. Mean voicekey RTs for naming the 248 pictures were available from two unpublished studies with 104 psychology undergraduates. In one study (Csapo, 1971), each of four sets of 65 slides was named by four different groups of 20 introductory psychology students. The pictures, shown at a rate of one every $15 \mathrm{sec}$, remained in view for $13.75 \mathrm{sec}$. The subjects had $10 \mathrm{sec}$ to respond. Voicekey RTs were also available from an unpublished 1974 study in which each of 24 subjects named 271 pictures, half in each of two sessions separated by 1 week. The subjects had $10 \mathrm{sec}$ to respond, interitem intervals varied with $\mathrm{RT}$, and different orders of trays and items within trays (forward or reverse) were used. The mean RTs from these two studies correlated .86, and they were averaged to produce one voicekey RT measure (VocRT) based on approximately 44 subjects per picture.

\section{Results and Discussion}

Mean RTs and standard deviations varied greatly across subjects, so individual $z$-scores were calculated, truncated at plus or minus three standard deviations to reduce the effects of extreme values, and then averaged. For each item, we initially computed a mean standardized RT for each of 16 cells defined by the four groups (NN, II, NI, IN), the two sessions $(1,2)$, and the two item orders. Order was confounded with equipment differences for the NI and IN groups, but correlations between the two orders were comparable for these mixed task groups and the same-task NN and II groups. Accordingly, the RTs were collapsed across order to produce four imaging and four naming means per item. Each mean was based on 24 RTs.

Reciprocity of naming and imaging. A principalcomponents factor analysis of the 8 means, followed by varimax rotation, indicated that two factors accounted for $78.38 \%$ of the variation among means. The rotated factor loadings shown in Table 1 clearly identified separate naming and imaging factors, which respectively accounted for $42.13 \%$ and $36.25 \%$ of the variation among the measures. On the basis of these results, we calculated a single manual naming mean (NmgRT) and a single manual imaging mean (ImgRT) for each item. ImgRT correlated .56 with NmgRT and .58 with the VocRT available from prior studies. The convergent validity of the keypress and 
Table 1

Study 1: Naming (N) and Imaging (I) Results for Sessions 1 and 2

\begin{tabular}{|c|c|c|c|c|c|c|c|c|}
\hline & \multicolumn{4}{|c|}{ Naming } & \multicolumn{4}{|c|}{ Imaging } \\
\hline & NNI & $\mathrm{NN} 2$ & NII & IN2 & III & II2 & INI & $\mathrm{NI} 2$ \\
\hline $\mathrm{NN}$ ! & & .86 & .90 & .90 & .49 & .47 & .50 & .51 \\
\hline NN2 & & & .79 & .80 & .44 & .43 & .42 & .48 \\
\hline NI1 & & & & .88 & .46 & .42 & .47 & .50 \\
\hline IN2 & & & & & .46 & 44 & .48 & .47 \\
\hline III & & & & & & .73 & .74 & .70 \\
\hline II 2 & & & & & & & .67 & .67 \\
\hline IN I & & & & & & & & .74 \\
\hline
\end{tabular}

\begin{tabular}{lllllllll}
\multicolumn{7}{c}{ Factor Loadings } \\
Naming & .92 & .82 & .88 & .89 & .25 & .25 & .27 & .30 \\
Imaging & .31 & .28 & .29 & .28 & .83 & .77 & .81 & .78 \\
& \multicolumn{7}{c}{ Cronbach's Alphas } \\
& .91 & .79 & .83 & .88 & .74 & .66 & .79 & .76 \\
\hline
\end{tabular}

Note-Letters indicate task and numbers session (e.g., NI2 denotes means for Session 2 of subjects naming in Session 1 and imaging in Session 2).

voicekey measures was confirmed by a correlation of .87 between NmgRT and VocRT. The average of NmgRT and standardized VocRT scores (NmgVcRT) correlated .59 with ImgRT. These correlations indicate that approximately one third of the variability across items in global imaging and naming RTs was shared.

Reliability. Additional analyses demonstrated that our naming and imaging RTs were consistent across subjects and stable over time, with RTs being more reliable for naming than for imaging. The averages of the Cronbach alpha coefficients of reliability in Table 1 were .85 for naming and .74 for imaging. The test-retest correlations for item means based on NN and II subjects were .86 for naming and .73 for imaging. The average correlations among the four means for each task were .86 for naming and .71 for imaging ( $r s$ in upper left and lower right triangles of Table 1).

Correlational estimates of the reciprocity between naming and imaging across the various conditions were also stable, as is shown in Table 1 . The 16 correlations between naming and imaging RTs ( $r$ in upper right square of Table 1) were all significant $(p s<.001)$ and averaged .47 , or .59 when corrected for attenuation using the mean same-task correlations to estimate reliabilities (McNemar, 1969, p. 171). We also calculated correlations between RTs for Sessions 1 and 2 individually for each subject. The means of the 24 correlations were .35 and .21 for the NN and II groups, and .14 and .11 for the NI and IN groups. Corrected for attenuation using the mean individual $r$ s for the NN and II groups, the latter values became .40 and .51 . The number of positive correlations out of 24 were $23,22,19$, and 20 for the NN, II, NI, and IN groups ( $p s<.025$ by a one-tailed sign test).

Complementing the correlational results, the mean RTs for naming $(1,097 \mathrm{msec})$ and imaging $(1,113 \mathrm{msec})$ in Session 1 were also similar in absolute terms. This finding is consistent with prior research (Tversky, 1969) and with the hypothesis that the two tasks involve reciprocal processes. Overall, the results demonstrated that a moder- ate relation exists between referential processing in the two directions, and justified a search for possible shared and unique predictors of naming and imaging RTs.

\section{PART 2: \\ PREDICTION OF NAMING AND IMAGING REACTION TIMES}

Dual coding theory and other similar interpretations of referential processing suggest a number of factors that should affect the ease of naming and imaging. Such factors as the number of different referential connections and their relative strengths would affect translation or crossover time, whereas such factors as the familiarity and complexity of the words and objects would affect the ease with which verbal and nonverbal representations are activated as stimuli during the initial phases of referential processing or as responses during the terminal phases.

\section{Predictors of Naming RT}

Name uncertainty refers theoretically to the number of distinct name codes to which images are connected; it can be measured by the number of different names given to a particular picture across subjects. Pictures that elicit many different names have higher uncertainty than those eliciting few names. Associative strength denotes a property of the individual connections between images and names, and can be measured by the percentage of subjects giving each name or the percentage of subjects repeating names on a second occasion. Although uncertainty and strength are theoretically distinct, the number of different names correlates highly with both the percentage correct names (Snodgrass \& Vanderwart, 1980) and naming stability (Butterfield \& Butterfield, 1977). As expected, picture-naming RTs increase with preexperimental uncertainty (Lachman, 1973; Lachman, Shaffer, \& Hennrikus, 1974), and they also increase with experimental uncertainty (Fraisse, 1964; Gholson \& Hohle, 1968; Morin, Konick, Troxell, \& McPherson, 1965) - that is, with the size of the item set from which stimuli are selected. Set size has less effect on the reading of words, which implies that the effects found for naming occur during referential translation from nonverbal to verbal symbolic modes.

Picture-naming RT also decreases as name frequency increases (Oldfield \& Wingfield, 1964, 1965), increases as age of word acquisition increases (Carroll \& White, 1973b), and decreases with practice (Fraisse, 1960, 1968), especially for unfamiliar pictures (Bartram, 1973; Oldfield \& Wingfield, 1964, 1965) and line drawings (Bartram, 1974). Although consistent with the assumption that the strengths of individual referential connections vary with experience, the loci of these familiarity, practice, and transfer effects in picture naming are ambiguous (e.g., see Bartram, 1974; Warren \& Morton, 1982), because such experiences undoubtedly affect more than the referential or crossover stage of processing. In particular, access to verbal representations that correspond to 
responses could depend on practice, familiarity, and perhaps other word characteristics (e.g., pronunciation difficulty). The picture-identification phase of naming could also be affected by analogous variables. That is, picture-naming RT might be affected by determinants of the ease of image activation, such as (1) picture familiarity and recency-although word frequency has only modest effects on picture-identification time (Wingfield, 1968); and (2) picture complexity, which has been shown to affect object perception (Long \& Wurst, 1984), the initial phase of naming.

\section{Predictors of Imaging RT}

Imaging RTs should be influenced by variables similar to those identified for naming. The number of different referents for a given word indicates the uncertainty involved in imaging and should therefore correlate negatively with imaging RT (Paivio, 1986, chap. 4), at least if image arousal parallels naming in this regard. Image uncertainty has not been investigated previously, but possible measures of image uncertainty include self-ratings of the number, typicality, and stability of images, and judged similarity of drawings across subjects or sessions. Ease of imaging also varies with the strength of the referential pathways from names to images, which in turn depend upon prior experiences. Word concreteness and imagery value (Paivio, 1974; Paivio, Yuille, \& Madigan, 1968) were originally developed as measures of the availability and strength of verbal-to-nonverbal referential connections. Both measures correlate with imaging RT, and with one another (e.g., see Janssen, 1976; Pavio, 1966, $1968,1975)$. The correlation was expected to be weaker in the present study because the range of concreteness and imagery was restricted to concrete words.

Theory and prior research suggest that attributes of the individual words and objects should also influence image generation. That is, imaging RTs should correlate with variables that reflect the ease of activating name codes during the perceptual phase (e.g., word frequency, pronunciation difficulty) and the ease of activating image codes during the response phase (e.g., object familiarity and complexity). Object complexity has correlated with imagery RT in some studies (e.g., Farah \& Kosslyn, 1981; Kosslyn, 1980), and has reliable effects on such related tasks as mental rotation (e.g., see Bethell-Fox \& Shepard, 1988; Folk \& Luce, 1987). However, complexity does not always influence image generation (Hoffmann, Denis, \& Ziessler, 1983). One possible explanation for the inconsistent results in generation tasks is that task demands influence image generation and might interact with complexity. Simpson and Paivio (1968) found, for example, that having to describe an image after a keypress resulted in slower latencies than a keypress alone. Like describing, drawing requires attention to detail and ought to be more sensitive to complexity effects than less demanding criteria for imagery are.

The following study included measures of uncertainty in the verbal-to-nonverbal direction and in the nonverbal- to-verbal direction, as well as measures related to the familiarity and complexity of verbal and nonverbal representations. A broad set of variables permitted us to determine their relative importance as predictors of naming and imaging, and to isolate effects of correlated variables. For example, object complexity correlates with such variables as familiarity (e.g., see Snodgrass \& Vanderwart, 1980) and perhaps even imagery value (Yuille, 1973). Factor analysis was used to relate naming and imaging RTs to orthogonal factors that represent the underlying processes more purely than do individual predictors, which tended to be complex and loaded on several factors.

\section{Method}

Subjects. The 31 predictors were based on (1) new ratings made by a total of 251 introductory psychology students; (2) the names or drawings generated by the $\mathbf{2 0 0}$ subjects described in Part 1; (3) additional names given by 122 psychology undergraduates; (4) available picture-familiarity ratings made by 45 introductory psychology students; and (5) published frequency and association counts. Except where noted otherwise, different subjects performed the various tasks. The subjects described in Part 1 also provided the mean imaging and naming RTs used as dependent variables.

General procedure. For the new ratings, the 248 words or pictures (black line drawings on white backgrounds) were presented in booklets in four different orders and preceded by four practice items. Table 2 lists the 31 variables, their abbreviations, and some descriptive statistics, including reliabilities for many of the measures. Correlations with available norms are presented in the text.

Nonverbal-to-verbal referential variables. Item means were calculated for the number of different names (DfrNm), percentage correct names (CorNm), and percentage missing names (Mis Nm). The means were averages of four scores based on the names given by the keypress group, by the two voicekey naming groups described in Part 1 , and by a total of 122 introductory psychology students who had $5 \mathrm{sec}$ to name each of one quarter of the individual pictures following 63-msec exposures (Csapo, 1971). The mean correlations among the four scores of each type appear in Table 2. For 114 of our items also in Snodgrass and Vanderwart (1980), their percentage target response score correlated .40 and -.50 with Cor Nm and Dfr Nm, respectively, and the sum of the omission categories correlated .30 with Mis Nm. The items common to the two sets contained our more consistently labeled items, which contributes to these low correlations. An additional 24 subjects rated number of names (NumNm) on a 7-point scale. A value of 1 indicated few names and 7 many. We also determined stability of names ( $\mathrm{StbNm}$ ) as the percentage of the $24 \mathrm{NN}$ subjects who gave the same name in both sessions.

Verbal-to-nonverbal referential variables. Drawings for the 72 keypress subjects who imaged in one or both sessions were classified by a judge as correct (i.e., corresponding to the target object), different from the target object, or missing. The number of different images (DfrIm) was determined by counting the number of distinct objects drawn, including the target. The percentage of missing images (MisIm) and percentage of correct images were calculated, but correct was omitted from the analysis because it correlated .98 with missing. DfrIm correlated .23 with Snodgrass and Vanderwart's (1980) number of nontarget images, and MisIm correlated .19 with their number of no-image responses (114 items). The low correlations partly reflect the consistency of imaging and the few omissions for these items. A judge rated similarity of Session 1 drawings for 8 random pairs of subjects on a 5-point scale where 5 indicated high similarity. The average of the 8 ratings produced a single measure of agreement of images (AgrIm). The 
Table 2

Summary of Measures

\begin{tabular}{|c|c|c|c|c|}
\hline \multicolumn{2}{|r|}{ Measure } & \multirow{2}{*}{$\frac{\text { Mean }}{3.33}$} & \multirow{2}{*}{$\begin{array}{l}S D \\
2.40\end{array}$} & \multirow{2}{*}{$\frac{\text { Reliability* }}{.76 \text { mean } r}$} \\
\hline DfrNm & Different names & & & \\
\hline CorNm & Correct names & 84.30 & 18.05 & .75 mean $r$ \\
\hline MisNm & Missing names & 1.48 & 2.91 & .41 mean $r$ \\
\hline NumNm & Rated number of names & 2.46 & .59 & .83 \\
\hline StbNm & Stability of naming & 89.05 & 12.05 & \\
\hline DfrIm & Different images & 1.45 & 1.35 & \\
\hline MisIm & Missing images & 3.23 & 6.92 & \\
\hline AgrIm & Agreement of drawn images & 3.75 & .52 & .71 \\
\hline StbIm & Stability of drawn images & 4.67 & .52 & .54 \\
\hline TypIm & Typicality of images & 5.26 & .63 & .76 \\
\hline CnsIm & Consistency of images & 5.55 & .57 & .63 \\
\hline NumIm & Number of images & 3.24 & .83 & .84 \\
\hline EasIm & Ease of imagery & 5.07 & .76 & .81 \\
\hline VivIm & Vividness of imagery & 5.54 & .77 & .85 \\
\hline PmWr & Pronunciation difficulty & 1.93 & .74 & .94 \\
\hline LenWr & Number of characters & 5.76 & 2.12 & \\
\hline SylWr & Number of syllables & 1.78 & .88 & \\
\hline FamWr & Familiarity of word & 4.97 & .87 & .92 \\
\hline AgeWr & Age at word acquisition & 4.72 & 1.38 & .96 \\
\hline KFrWr & Kucera-Francis & 1.01 & .71 & \\
\hline ThLWr & Thorndike-Lorge & 1.27 & .61 & \\
\hline SpkWr & Spoken frequency of word & .31 & .44 & \\
\hline AvlWr & Availability of word & 1.82 & .93 & \\
\hline $\mathrm{CmpPc}$ & Complexity of picture & 3.42 & 1.25 & .96 \\
\hline Cmplm & Complexity of image & 3.21 & 1.00 & .93 \\
\hline DrwIm & Drawing time for image & 9.52 & 3.13 & $.85 .89 \dagger$ \\
\hline Realm & Realism of image & 5.29 & .79 & .83 \\
\hline Piclm & Picture-image similarity & 5.40 & .78 & .85 \\
\hline FamPc & Familiarity of picture & 4.59 & 1.42 & \\
\hline $\operatorname{RecPc}$ & Recency from picture & 4.01 & 1.51 & .94 \\
\hline RecIm & Recency from imagery & 3.79 & 1.32 & .94 \\
\hline
\end{tabular}

*Coefficient alpha unless stated otherwise. †Alpha for Sessions 1 and 2.

same judge rated similarity for paired Session 1 and 2 drawings for $\mathbf{8}$ subjects from the II condition as a measure of stability of images (StbIm).

Typicality of images (TypIm) was rated by 25 subjects, with a rating of 1 indicating an uncommon and 7 a typical image. After rating image vividness (see below), 25 subjects rated the words a second time on consistency of imagery (CnsIm), defined as the similarity between their first and second images. A 1 indicated a very different or variable image and a 7 a very consistent image. Number of images (NumIm) was rated by 25 subjects, with 1 indicating few or no images and 7 many images. Ease of imagery (EasIm) was rated by 24 subjects on a 7-point scale, where 1 indicated "difficult" and 7 "easy to image." Another 26 subjects rated vividness of imagery (VivIm), where 1 indicated "not at all clear" or "no image" and 7 indicated "very clear and vivid." Ease of imagery ratings from Paivio (1974) correlated .50 and .53 (115 items) with EasIm and VivIm-respectable correlations given the restricted range of imagery values.

Verbal attributes. Word complexity was measured in several ways. Difficulty of word pronunciation (PrnWr) was rated by 24 introductory psychology students, with a rating of 1 representing easy-to-pronounce words and 7 difficult-to-pronounce words. The mean ratings correlated .90 (228 items) with unpublished ratings from 46 introductory psychology students, each of whom rated about half of 649 words. We also determined length of word (LenWr) in characters, and syllables per word (SylWr).

Six measures were related to word familiarity and frequency. The familiarity of words (FamWr) in print was rated by 25 subjects on a 7-point scale, with 7 indicating "very familiar." The mean ratings correlated .88 (216 items) with familiarity ratings from Pai- vio (1974). Age of word acquisition (AgeWr) was rated by 24 subjects on a 9-point scale, where 1 referred to the pre-nursery school period at age 2, and 9 referred to Grade 8 or later. Mean ratings correlated .90 (90 items) with Carroll and White (1973a) and .93 (166 items) with unpublished ratings of 424 concrete and 171 abstract words each rated by about 40 undergraduates at Western (total $N=161$ ). Log KuČera-Francis frequency (KFrWr; see Kucera \& Francis, 1967), log Thorndike-Lorge frequency (ThLWr), and, from Brown (1984), log spoken frequency (SpkWr) were determined for each word. We also calculated log availability of the words (AvlWr) from the number of subjects giving each word as a response in the Kiss, Armstrong, and Milroy (1972) association norms (cf. Rubin \& Friendly, 1986). To remove zeros, one was added to raw frequencies before these logs (base 10) were calculated.

Nonverbal attributes. Complexity of pictures $(\mathrm{CmpPc})$ was rated by 27 subjects. Complexity was defined in terms of number of parts, with 1 representing low complexity or simple objects and 7 high complexity. The complexity of images (CmpIm) of the objects was rated on the same scale by 27 subjects given words as stimuli. The picture complexity ratings of Snodgrass and Vanderwart (1980) correlated .73 with $\mathrm{CmpPc}$ and .77 with $\mathrm{CmpIm}$ (114 items). As another measure of complexity, we measured individual drawing times for 12 subjects in the II condition (cf. Yuille, 1973). The mean drawing times for Sessions 1 and 2 correlated .91 , so a single mean image drawing time (DrwIm) was calculated for each item. Realism of images (ReaIm) was rated by 24 subjects, with 1 indicating "artificial" or "not at all realistic" and 7 indicating "very realistic and true to life." These same subjects then rated the similarity of their prior images to our pictures on a picture-image similarity (PicIm) scale, with 7 indicating "very similar." 
We obtained several ratings of picture familiarity similar to those for words, but objective counts analogous to word frequency are not available for nonverbal material. Csapo (1971; Paivio, 1973) had 45 introductory psychology students rate familiarity of pictures (FamPc), with 7 representing familiar objects. The mean ratings correlated .91 (114 items) with the mean picture familiarity ratings of Snodgrass and Vanderwart (1980). The 24 subjects who rated number of different names subsequently rated recency from pictures $(\mathrm{RecPc})$. A value of 1 indicated that they had experienced the object either never or a very long time ago, and 7 , very recently. The subjects who rated number of images later rated recency from images (RecIm), given only the names.

\section{Results and Discussion}

Correlations among the 31 predictors are presented in the Appendix, and their correlations with imaging and naming RTs in Table 3. As is shown in Table 3, many of the variables correlated substantially with imaging or naming RTs, or both. Because predictors also correlated highly with one another (see Appendix), the relationships between referential-processing RTs and the 31 predictors were examined by factor analyses of the RTs and predictors together. Factor analysis also permitted us to allocate the correlation between naming and imaging RTs to different factors, since the sum across factors of the products of RT loadings equals the correlation between naming and imaging RTs. The reported analysis is based on NmgVcRt, the average of the NmgRTs and stan-

Table 3

Correlation of Individual Predictors and Reaction Times

\begin{tabular}{|c|c|c|c|c|}
\hline & \multicolumn{4}{|c|}{ RT Measure } \\
\hline & ImgRT & NmgVcRT & NmgRT & VocRT \\
\hline MisNm & 46 & 78 & 67 & 79 \\
\hline MisIm & 53 & 59 & 52 & 59 \\
\hline DfrIm & 28 & 31 & 22 & 33 \\
\hline AgeWr & 70 & 60 & 55 & 59 \\
\hline DfrNm & 58 & 84 & 77 & 84 \\
\hline CorNm & -53 & -73 & -64 & -74 \\
\hline StbNm & -27 & -46 & -44 & -45 \\
\hline PicIm & -37 & -52 & -55 & -49 \\
\hline Cnsim & -28 & -21 & -18 & -22 \\
\hline Typlm & -50 & -44 & -42 & -43 \\
\hline ReaIm & -57 & -49 & -43 & -49 \\
\hline VivIm & -63 & -53 & -50 & -52 \\
\hline EasIm & -66 & -58 & -53 & -58 \\
\hline FamWr & -66 & -47 & -41 & -47 \\
\hline KFrWr & -54 & -30 & -27 & -30 \\
\hline SpkWr & -37 & -23 & -19 & -23 \\
\hline NumIm & -50 & -36 & -33 & -36 \\
\hline NumNm & -14 & -03 & -04 & -03 \\
\hline AvlWr & -61 & -36 & -34 & -36 \\
\hline ThLWr & -63 & -41 & -38 & -40 \\
\hline PrnWr & 63 & 37 & 33 & 37 \\
\hline LenWr & 51 & 18 & 16 & 18 \\
\hline SylWr & 49 & 23 & 21 & 23 \\
\hline FamPc & -46 & -31 & -29 & -30 \\
\hline $\operatorname{RecPc}$ & -44 & -28 & -28 & -27 \\
\hline RecIm & -47 & -21 & -19 & $-2 I$ \\
\hline $\mathrm{CmpPc}$ & 18 & 01 & 05 & -01 \\
\hline Cmplm & 27 & 07 & 09 & 06 \\
\hline DrwIm & 22 & -06 & -01 & -08 \\
\hline Agrlm & -28 & -22 & -26 & -20 \\
\hline Stblm & -16 & -10 & -12 & -09 \\
\hline
\end{tabular}

Table 4

Factor Loadings for Reaction Times and Predictors (Decimals Omitted)

\begin{tabular}{|c|c|c|c|c|c|c|c|}
\hline & & & & \multicolumn{4}{|c|}{ Factors } \\
\hline & \multicolumn{3}{|c|}{ Referential } & \multicolumn{2}{|c|}{ Verbal } & \multicolumn{2}{|c|}{ Nonverbal } \\
\hline & $\mathrm{Com}$ & Nmg & Img & Fam & $\mathrm{Cmp}$ & Fam & Cmp \\
\hline $\begin{array}{l}\text { Image RT } \\
\text { NmgVc RT }\end{array}$ & $\begin{array}{l}-42 \\
-84\end{array}$ & $\begin{array}{l}-17 \\
-35\end{array}$ & $\begin{array}{l}-32 \\
-07\end{array}$ & $\begin{array}{l}-37 \\
-16\end{array}$ & $\begin{array}{l}-47 \\
-15\end{array}$ & $\begin{array}{l}-18 \\
-14\end{array}$ & $\begin{array}{l}-21 \\
-04\end{array}$ \\
\hline MisNm & $-80^{*}$ & -10 & -12 & -18 & -06 & -01 & 03 \\
\hline MisIm & $-59 *$ & -03 & $-41^{*}$ & -24 & -18 & -04 & -03 \\
\hline DfrIm & $-40^{*}$ & -04 & -18 & -03 & 02 & -01 & -01 \\
\hline AgeWr $_{r}$ & $-46^{*}$ & -07 & -30 & $-45^{*}$ & $-41^{*}$ & -24 & 08 \\
\hline DfrNm & $-66^{*}$ & $-61^{*}$ & -19 & -14 & -11 & -10 & -01 \\
\hline CorNm & $52 *$ & $68^{*}$ & 20 & 21 & 16 & 01 & -06 \\
\hline StbNm & 28 & $69 *$ & 17 & 04 & 01 & -06 & 02 \\
\hline PicIm & $41^{*}$ & 26 & $44^{*}$ & -05 & -14 & 26 & 17 \\
\hline CnsIm & 16 & 12 & $69^{*}$ & -02 & -14 & 02 & 18 \\
\hline TypIm & 33 & 14 & $62^{*}$ & 17 & -03 & 31 & 16 \\
\hline Realm & 38 & 06 & $59 *$ & 18 & 12 & 38 & -05 \\
\hline VivIm & 39 & 08 & $65^{*}$ & $40^{*}$ & 08 & 31 & 18 \\
\hline EasIm & $52^{*}$ & 00 & $45^{*}$ & $52^{*}$ & 15 & 17 & -02 \\
\hline FamWr & 30 & -00 & 38 & $68^{*}$ & 25 & 36 & -00 \\
\hline $\mathrm{KFrWr}$ & 04 & 12 & 02 & $81^{*}$ & 29 & 25 & 03 \\
\hline$S_{p k} W_{r}$ & 03 & 15 & -06 & $71^{*}$ & 15 & 22 & 04 \\
\hline NumIm & 31 & -14 & 11 & $74^{*}$ & 13 & 14 & -05 \\
\hline NumNm & 12 & -39 & 08 & $47^{*}$ & -05 & -03 & 02 \\
\hline AvlWr & 12 & 12 & 17 & $77^{*}$ & $40^{*}$ & 12 & -03 \\
\hline ThlWr & 16 & 12 & 09 & $70 *$ & $41^{*}$ & 17 & 01 \\
\hline PrnWr & -19 & -02 & -09 & -38 & $-82^{*}$ & -13 & 01 \\
\hline LenWr & -01 & -03 & 08 & -29 & $-83^{*}$ & -03 & 03 \\
\hline SylWr & -06 & -06 & 11 & -24 & $-82^{*}$ & -06 & 00 \\
\hline FamPc & 12 & -00 & 22 & 25 & 13 & $84 *$ & 22 \\
\hline $\operatorname{RecPc}$ & 10 & 01 & 15 & 34 & 07 & $83 *$ & 21 \\
\hline RecIm & 03 & -04 & 22 & $44^{*}$ & 16 & $74 *$ & 20 \\
\hline $\mathrm{CmpPc}$ & 07 & 00 & -02 & 15 & -16 & -23 & $-80^{*}$ \\
\hline CmpIm & 01 & 06 & 07 & 14 & -39 & -26 & $-68^{*}$ \\
\hline DrwIm & 14 & 03 & -03 & -07 & -01 & -19 & $-85^{*}$ \\
\hline AgrIm & 22 & 05 & 18 & 09 & -14 & -02 & $68^{*}$ \\
\hline StbIm & 07 & 04 & 19 & 08 & -21 & -05 & $67^{*}$ \\
\hline \% Variation & 13.0 & 5.6 & 9.0 & 15.7 & 10.3 & 9.0 & 9.3 \\
\hline Total \% & 71.9 & & & & & & \\
\hline
\end{tabular}

*Predictor loadings of .4 or greater.

dardized VocRTs described in Part 1, but similar results were obtained when the imaging RTs and the separate naming RTs were analyzed.

Table 4 presents a 7 -factor solution obtained by principal factor analysis with iteration followed by varimax rotation. The decision to report 7 factors was based on ease of interpretation, on the fact that 6 to 8 factors had eigenvalues greater than one in various analyses, and on other considerations. In particular, the proportion of variability in NmgVcRT accounted for by the factors was .74, .90 , and .90 for the $6-, 7-$, and 8 -factor solutions. The communality for ImgRT was .74 in all three cases. Moreover, the maximum loading on the eighth factor was only .26 , whereas the last factor in the 7 -factor solution had loadings greater than .60. The 7-factor solution was also very stable across items. Loadings from the overall analysis correlated highly with comparable loadings from separate analyses of odd and even items $(r s=.97$, across 231 loadings). Corresponding odd and even loadings also correlated highly $(r=91)$. Consistency across subjects 
was confirmed by high correlations between the 14 RT loadings in the overall analysis and comparable loadings in separate analyses of the mean male and female RTs ( $r$ s $=.95$ and .94 , respectively), which also correlated highly $(r=.98)$.

The rotated factor analysis accounted for $71.9 \%$ of the variation in the scores, and the variance accounted for by each factor is shown in Table 4 . The factor loadings for RTs are all negative, so that positive loadings for predictors reflect faster RTs and negative loadings for predictors reflect slower RTs. In the text, $(-)$ indicates predictors with negative loadings.

Referential factors. Three factors had high loadings for variables related to referential translation between the verbal and nonverbal systems and accounted for much of the variation in RTs. One of these factors contained variables related to both naming and imaging, and the others contained variables more specific to naming and imaging.

Common referential. The common referential factor was related to both naming and imaging, including substantial negative loadings for missing names and images, and different names and images. Variables with positive loadings were picture-image similarity, correct names, and ease of imagery. RTs for both naming and imaging, but especially naming, were slower with increases in missing names, missing images, different names, and different images. RTs were faster as correct names, pictureimage similarity, and ease of imagery increased. As measured by squared factor loadings, this factor accounted for $17 \%$ of the variation in imaging RTs, $71 \%$ of the variation in naming RTs, and a substantial $59.4 \%$ of the correlation between imaging and naming RTs-that is, $59.4=100 \times(.42 \times .84) / .59$, where .42 and .84 are the ImgRT and NmgVcRT factor loadings, and .59 is the $r$ between ImgRT and NmgVcRT.

Referential naming. As well as contributing to the common factor, different names ( - ) and correct names loaded on a specific naming factor, which suggests that these variables reflect several facets of naming. This factor also included stability of names and rated number of different names ( - ), two variables not loading on the common factor. That this factor had high loadings for rated and actual number of different names, as well as name stability, suggests that name uncertainty may be the underlying mechanism. The referential naming factor predicted $12 \%$ of the variation in naming RT and 3\% of the variation in imaging RT, and it accounted for $9.8 \%$ of the correlation between RTs. Naming RTs in particular became slower as name uncertainty increased.

Referential imagery. A unique imagery factor was identified by such variables as the consistency, typicality, realism, and vividness of images. Several imagery variables loaded on both the common referential factor and this imagery factor, notably percent missing images (-), picture-image similarity, and rated ease of imagery. The referential imagery factor accounted for $10 \%$ of the variation in imaging RT, only $2 \%$ of the variation in naming RT, and $3.7 \%$ of the correlation between the two. Although image consistency and typicality suggest uncer- tainty as a possible explanation for the decrease in imagery RTs, other variables designed to tap image uncertainty had only weak loadings on this factor and sometimes loaded in the wrong direction. Number of different images had a negative loading, as expected, but the (weak) loadings for rated number and agreement of images, as well as image stability judged from the drawings, were positive. Such findings suggest that uncertainty may not have the same effects on imaging as on naming, but this conclusion is tempered by our inadequate understanding of such measures as rated number of images and judged agreement of drawings.

Stimulus and response factors. The remaining factors were identified as verbal familiarity, verbal complexity, nonverbal familiarity, and nonverbal complexity. These factors define characteristics of the individual stimuli and responses, and were generally stronger predictors of imaging RTs than of naming RTs.

Verbal familiarity. This factor was defined primarily by printed familiarity ratings, Kučera-Francis (1967) and the other two frequency measures, and availability in the Kiss norms. Rated number of images and names, especially the former, also loaded more on word familiarity than on the referential uncertainty factors they were designed to tap. Secondary loadings for age of acquisition (-) and difficulty of pronunciation $(-)$ are consistent with a verbal familiarity interpretation, whereas loadings for ease of imagery and recency of experience of the imagined object suggest a possible influence of nonverbal processes. Verbal familiarity accounted for $13.6 \%$ of the variation in imaging RTs, $2.5 \%$ of the variation in naming RTs, and $9.8 \%$ of their correlation. This variable is presumed to affect the ease of activation of verbal codes, before the referential phase in imaging and after it in naming.

Verbal complexity. Dominant negative loadings for rated difficulty of pronunciation, length of word, and number of syllables defined this factor. Modest secondary loadings for age of word acquisition (-), ThorndikeLorge frequency, and availability suggest that one's prior experience of words facilitates their pronunciation. The secondary loading for complexity of images $(-)$ suggests either that some raters confused object and word complexity or that word complexity directly interfered with image generation. Verbal complexity accounted for a substantial $22.2 \%$ of the variation in imaging RT, $2.1 \%$ of the variation in naming RT, and $11.7 \%$ of their correlation. The modest effect on naming possibly reflects response generation processes. The effect of verbal complexity on imaging seems too strong to reflect only the activation of verbal representations prior to imaging, suggesting that verbal complexity may have produced visual interference (cf. Brooks, 1967). That is, because stimuli remained visible until a response occurred, subjects may have had difficulty generating visual images when long, complex words were shown.

Nonverbal familiarity. This factor was defined by dominant loadings for picture familiarity and recency of experience of the pictured or imaged object. A secondary 
loading for word familiarity indicates some relation between word and object familiarity. Moderate secondary loadings for realism, typicality, and vividness of images suggest that such attributes may depend partly on familiarity or recency of experience of the corresponding objects. Nonverbal familiarity accounted for $3.1 \%$ of the variation in imaging RT, $1.8 \%$ of the variation in naming RT, and $4.1 \%$ of their correlation. These modest effects of nonverbal familiarity could reflect its effects on ease of activation of the image, either on the stimulus side of naming or on the response side of imaging.

Nonverbal complexity. The major defining variables were drawing time and rated complexity of pictures or images, all with negative loadings. That judged agreement and stability of the drawings also loaded primarily on this factor indicates that simple objects were judged to be drawn more consistently across subjects and sessions than were complex objects. Imaging RTs decreased with decreases in nonverbal complexity, which accounted for $4.6 \%$ of the variation in imaging $\mathrm{RT}, 0.2 \%$ of the variation in naming RT, and only $1.5 \%$ of their correlation. The effect of nonverbal complexity on imaging is consistent with the findings of Kosslyn (1980). The effect might reflect image construction processes, as postulated by Kosslyn, but it could also be due to processes after image generation. That is, even images generated in parallel might involve serial processes sensitive to complexity when attention to detail is required, as would be the case in drawing.

\section{Summary of Study 2}

Our analysis accounted for $89.7 \%$ of the variation in naming RTs, with the common referential and referential naming factors being particularly strong predictors. Verbal familiarity, verbal complexity, and nonverbal familiarity made smaller contributions. The factor analysis accounted for $73.8 \%$ of the variation in imaging RTs, which had sizable loadings on four of the seven factors. As expected, a common referential factor and a referential imagery factor contributed to the prediction of imaging RTs, as did verbal familiarity and verbal complexity. Smaller contributions were made by referential naming, nonverbal familiarity, and nonverbal complexity. The correlation between naming and imaging RTs was largely explained by their mutual loadings on the common referential factor, with smaller shared effects of the referential naming, verbal familiarity, and verbal complexity factors.

\section{GENERAL DISCUSSION}

Naming and imaging activities appear to be mediated by common and distinct structural and functional mechanisms that can be inferred from the factor pattern. The common referential factor had large loadings for missing names and missing images, suggesting that the avail- ability of bidirectional or reciprocal referential connections may be the mechanism responsible for much of the correspondence between naming and imaging. That is, nonexistent or weak connections in one direction appear to be associated with nonexistent or weak connections in the other direction. Components of different names and different images also contributed to this common factor, as would be expected if diverse, weak responses occur as subjects attempt to name or image items that lack stable referential connections. The emergence of this common factor is consistent with our earlier suggestion that referential processing experiences normally strengthen connections in both directions. This hypothesis was based on a dual coding analysis, but similar explanations presumably could be proposed by common coding theorists who postulate a more complex system in which naming and imaging both involve two steps, one from the stimulus representation to an abstract conceptual representation and the other from the latter to the response code. Exactly how such a system would operate to account for the present data remains to be specified.

The other factor that contributed substantially to naming RTs was the referential naming factor, which included several measures of name uncertainty. In particular, naming RTs slowed dramatically as number of different names increased, although this variable loaded on both the common and naming referential factors. The effect of uncertainty is consistent with prior research by Lachman (1973), and with evidence that uncertainty and response competition disrupt picture naming among aphasics (e.g., Mills, Knox, Juola, \& Salmon, 1979; Rochford \& Williams, 1962). At least two theoretical mechanisms could explain why naming slows down as the number of competing responses increases: (1) activation may be less vigorous when diffused over multiple pathways, or (2) competing names may inhibit one another and thereby delay the emergence of a particular response. Weak or modest loadings for naming RTs on the remaining factors indicate that stimulus and response factors had little effect under the conditions of the present study, and that the referential imaging variables were unrelated to naming except as reflected in the common referential factor.

Imaging RTs loaded substantially on the common referential factor, which can be explained by means of processes similar to those used to explain naming RTs. That is, weak referential connections in one direction are associated with weak connections in the reverse direction. Imaging also correlated with the unique referential imaging factor, which included several variables related to image uncertainty (consistency and typicality of imagery). As expected, imaging RTs increased as number of different images increased; but the correlation was much weaker than in the case of naming, and, contrary to the uncertainty hypothesis, imaging got faster as rated number of different images increased. The larger and more consistent effect of name than of image uncertainty could sim- 
ply represent differences in the quality of the measures, but the distinct properties of the imagery and verbal systems (Paivio, 1986) could also contribute to their differing uncertainty effects. That is, the continuous, analogue quality of imagery means that with images, unlike words, there is no clear boundary between one response and another, and the parallel nature of imagery means that image uncertainty might generate less mutual interference than would be the case for verbal representations that are processed serially.

Unlike naming, much of the variation in imaging was predicted by nonreferential factors. The stimulus attributes of verbal familiarity and verbal complexity were particularly important. Verbal familiarity presumably affects the ease of activating the word code that drives subsequent processing in the imaging task. However, a number of the variables loading on this factor implicated nonverbal processes and suggest a more complex story. The pattern of results suggests that experience contributes in complex ways to the development of verbal and imaginal representations and their interconnections, and additional research will be needed to tease apart the subtle relations among word and object familiarity, and imagery variables.

The robust effect of verbal complexity on imaging RTs was unexpected, but the effect is consistent with previous research on modality-specific interference (e.g., Brooks, 1967) if we assume further that the amount of interference varies with visual word complexity. Such interference would not be expected in the case of naming, because visual processing of pictures would not compete with the nonvisual naming activity.

The response factors of nonverbal familiarity and nonverbal complexity had small but reliable effects on imaging. The effect of nonverbal complexity is consistent with the findings of Kosslyn (1980) rather than those of Hoffmann et al. (1983), perhaps because drawing required attention to details in the same way as episodic memory demands might have done in Kosslyn's studies. The effect of nonverbal familiarity on imaging is new; but it can be accounted for in terms of the availability of the underlying imaginal representation, and it parallels earlier research showing that verbal familiarity facilitates naming (e.g., Oldfield \& Wingfield, 1964, 1965).

With respect to the reciprocity of naming and imaging, the correlation between imaging and naming RTs and their mutual relations with some shared predictors are consistent with earlier research on individual differences (Bucci, 1984) and transfer effects (Thompson et al., 1986). The hypothesis that people who are slow at imaging are also slow at naming was confirmed by supplementary analyses, which showed strong correlations between mean naming and imaging RTs for IN and NI subjects (mean $r=.71$ ). However, individual differences in global RT undoubtedly contributed to that correlation, making it less uniquely attributable to referential processing than to correlations across items. Further research is needed to determine whether the correlation between naming and imaging across people, as well as transfer between naming and imaging tasks, varies with item attributes such as those identified in this paper. Several analyses did show that item attributes interacted with individual differences. For example, number of different names had a stronger effect on naming times for slow than fast subjects. Such effects might reflect individual differences with respect to the predictors (e.g., recency of experience), or subjects may vary systematically in how sensitive they are to the effects of different variables.

In conclusion, the present research has demonstrated the value of studying referential processing from a multivariate perspective under the guidance of a theory that explicitly recognizes the reciprocity between naming and imaging. Prior research and dual coding theory led to the generation of a rich collection of variables that were meaningfully related to response times in one or both of the referential processing tasks. The benefit of simultaneously considering referential processing in both directions was seen in the effects on both naming and imaging of missing images, ease of imagery, and other variables that might have been expected intuitively to affect referential processing in only one direction. Moreover, many of the measures were factorially complex, and the multivariate approach was essential to identifying simple, and sometimes contrasting, components that could be related to RTs in meaningful ways. Many such complexities remain to be investigated and resolved by further theoretically motivated multivariate and, ultimately, experimental studies.

\section{REFERENCES}

BarTRAM, D. J. (1973). The effects of familiarity and practice on naming pictures of objects. Memory \& Cognition, 1, 101-105.

BARTRAM, D. J. (1974). The role of visual and semantic codes in object naming. Cognitive Psychology, 6, 325-356.

BETHELl-Fox, C. E., \& SHEPARD, R. N. (1988). Mental rotation: Effects of stimulus complexity and familiarity. Journal of Experimental Psychology: Human Perception \& Performance, 14, 12-13.

Brooks, L. R. (1967). The suppression of visualization by reading. Quarterly Journal of Experimental Psychology, 19, 289-299.

Broverman, D. M. (1960). Cognitive style and intra-individual variation in abilities. Journal of Personality, 28, 240-256.

Brown, G. D. A. (1984). A frequency count of 190,000 words in the London-Lund Corpus of English Conversation. Behavior Research Methods, Instruments, \& Computers, 16, 502-532.

Brown, W. (1915). Practice in associating color-names with colors. Psychological Review, 22, 45-55.

Buccl, W. (1984). Linking words and things: Basic processes and individual variation. Cognition, 17, 137-153.

Bucci, W., \& Freeman, N. (1978). Language and hand: The dimension of referential competence. Journal of Personality, 46, 594-622.

Butterfield, G. B., \& Butterfield, E. C. (1977). Lexical codability and age. Journal of Verbal Learning \& Verbal Behavior, 16, 113-118.

Carroll, J. B., \& Wite, M. N. (1973a). Age-of-acquisition norms for 220 picturable nouns. Journal of Verbal Learning \& Verbal Behavior, 12, 563-576.

Carroll, J. B., \& White, M. N. (1973b). Word frequency and age 
of acquisition as determiners of picture-naming latency. Quarterly Journal of Experimental Psychology, 25, 85-95.

CATTELL, J. M. (1886). The time it takes to see and name objects. Mind, $11,63-65$

CSAPO, K. (1971). Stimulus attributes, presentation rate and coding in shor term memory. Unpublished doctoral dissertation, University of Western Ontario, London, Canada.

FARAH, M. J., \& KossLYN, S. M. (1981). Structure and strategy in image generation. Cognitive Science, 4, 371-383.

FoLK, M. D., \&uCE, R. D. (1987). Effects of stimulus complexity on mental rotation rate of polygons. Journal of Experimental Psychology: Human Perception \& Performance, 13, 395-404.

Fraisse, P. (1960). Recognition time measured by verbal reaction to figures and words. Perceptual \& Motor Skills, 11, 204.

Fraisse, P. (1964). Le temps de réaction verbale. Année Psychologique, 64, 21-46.

FRAISSE, P. (1968). Motor and verbal reaction times to words and draw ings. Psychonomic Science, 12, 235-236.

GHolson, B., \& HoHLe, R. H. (1968). Verbal reaction times to hues vs hue names and forms vs form names. Perception \& Psychophysics, 3, 191-196.

Hoffman, J., Denis, M., \& Ziessler, M. (1983). Figurative features and the construction of visual images. Psychological Research, 45 , $39-54$.

JANSSEN, W. (1976). On the nature of the mental image. Soesterberg, The Netherlands: Institute for Perception.

Kiss, G. R., Armstrong, C. A., \& Milroy, R. (1972). An associative thesaurus of English [microfilm]. Wakefield: E. P. Microforms.

KossLYN, S. M. (1980). Image and mind. Cambridge, MA: Harvard University Press.

KossLYN, S. M. (1987). Seeing and imaging in the cerebral hemispheres A computational approach. Psychological Review, 94, 148-175.

KuČERA, H., \& FrANCIS, W. N. (1967). Computational analysis of present-day American English. Providence, RI: Brown University Press.

LACHMAN, R. (1973). Uncertainty effects on time to access the inter nal lexicon. Journal of Experimental Psychology, 99, 199-208.

Lachman, R., Lachman, J. L. (1980). Picture naming: Retrieval and activation of long-term memory. In L. W. Poon, J, L. Fozard, L. S. Cermak, D. Arenberg, \& L. W. Thompson (Eds.), New direc tions in memory and aging (pp. 313-343). Hillsdale, NJ: Erlbaum.

Lachman, R., Shaffer, J. P., \& Hennrikus, D. (1974). Language and cognition: Effects of stimulus codability, name-word frequency, and age of acquisition on lexical reaction time. Journal of Verbal Learning \& Verbal Behavior, 13, 613-625.

Lazarus, R. S., Baker, R. W., Broverman, D. M., \& Mayer, J. (1956). Personality and psychological stress. Journal of Personality, 25, 559-577.

LoNG, G. M., \& WURST, S. A. (1984). Complexity effects on reactiontime measures of visual persistence: Evidence for peripheral and centra contributions. American Journal of Psychology, 97, 537-561.

McNemar, Q. (1969). Psychological statistics (4th ed.). New York: Wiley.

Mills, R. H., Knox, A. W., Juola, J. F., \& Salmon, S. J. (1979) Cognitive loci of impairments in picture naming by aphasic subjects Journal of Speech \& Hearing Research, 22, 73-87.

MOORE, T. V. (1915). The temporal relations of meaning and imagery. Psychological Review, 22, 177-225.

Morin, R. E., Konick, A., Troxell, N., \& McPherson, S. (1965). Information and reaction time for naming responses. Journal of $E x$ perimental Psychology, 70, 309-314.

MorTon, J. (1969). Interaction of information in word recognition. Psychological Review, 76, 165-178.

MorTon, J. (1979). Facilitation in word recognition: Experiments caus- ing change in the logogen model. In P. A. Kolers, M. Wrolstead. \& H. Bouma (Eds.), Processing of visible language (Vol. 1, pp. 259. 268). New York: Plenum.

OldFiELD, R. C., WingField, A. (1964). The time it takes to name an object. Nature, 202, 1031-1032.

OLDFELD, R. C., \& WINGFIELD, A. (1965). Response latencies in naming objects. Quanerly Journal of Experimental Psychology, 17, 273281

Paivio, A. (1966). Latency of verbal associations and imagery to noun stimuli as a function of abstractness and generality. Canadian Jour nal of Psychology, 20, 378-387.

Paivio, A. (1968). A factor-analytic study of word attributes and verbal learning. Joumal of Verbal Learning \& Verbal Behavior, 7, 41-49 Palvio, A. (1971). Imagery and verbal processes. New York: Holt. Rinehart \& Winston. (Reprinted 1979, Hillsdale, NJ: Erlbaum)

Paivio, A. (1973). [List of 260 pictures and their labels with normative data on selected attributes]. Unpublished raw data, Department of Psychology, University of Western Ontario, London, Canada.

Palvio, A. (1974). [Imagery and familiarity ratings for 2448 words] Unpublished norms, Department of Psychology, University of Western, Ontario, London, Canada.

Paivio, A. (1975). Neomentalism. Canadian Journal of Psychology, 29, 263-291.

Paivio, A. (1986). Mental representations: A dual-coding approach New York: Oxford University Press

Paivio, A., Yuille, J. C., \& Madigan, S. (1968). Concreteness, imagery, and meaningfulness values for 925 nouns. Journal of Experimental Psychology Monographs, 76(1, Pt. 2).

Potter, M. C., \& Faulconer, B. A. (1975). Time to understand pic tures and words. Nature, 253, 437-438.

Rochford, G., \& Williams, M. (1962). Studies in the development and breakdown of the use of names. Joumal of Neurology, Neurosurgery, \& Psychiatry, 225, 222-227, 228-233

Rubin, D. C., \& Friendly, M. (1986). Predicting which words get recalled: Measures of free recall, availability, goodness, emotionality, and pronunciability for 925 nouns. Memory \& Cognition, 14, 79-94.

Scarborough, D. L., Cortese, C., \& Scarborough, H. S. (1977). Frequency and repetition effects in lexical memory. Journal of Experimental Psychology: Human Perception \& Performance, 3, 1-17.

SEYmour, P. H. K. (1973). A model for reading, naming, and comparison. British Journal of Psychology, 64, 35-49.

Simpson, H. M., \& PaIvio, A. (1968). Effects on pupil size of manual and verbal indicators of cognitive task fulfillment. Perception \& Psychophysics, 3, 185-190.

Snodgrass, J. G. (1984). Concepts and their surface representations. Journal of Verbal Learning \& Verbal Behavior, 23, 3-22.

SnOdGrass, J. G., \& Vanderwart, M. (1980). A standardized set of 260 pictures: Norms for name agreement, image agreement, familiarity, and visual complexity. Joumal of Experimental Psychology: $\mathrm{Hu}$ man Learning \& Memory, 6, 174-215.

Thompson, C. K., Hall, H. R., \& Sison, C. E. (1986). Effects of hypnosis and imagery training on naming behavior in aphasia. Brain \& Language, 28, 141-153.

THORNDIKE, E. L., \& LORGE, I. (1944). The teacher's word book of 30,000 words. New York: Teachers' College, Columbia University.

TVersKy, B. (1969). Pictorial and verbal encoding in a short-term memory task. Perception \& Psychophysics, 6, 225-233.

WARREN, C., \&ORTON, J. (1982). The effects of priming on picture recognition. British Journal of Psychology, 73, 117-129.

WINGFIELD, A. (1968). Effects of frequency on identification and naming of objects. American Journal of Psychology, 81, 226-234.

YuiLle, J. C. (1973). A detailed examination of mediation in PA learning. Memory \& Cognition, 1, 333-342. 


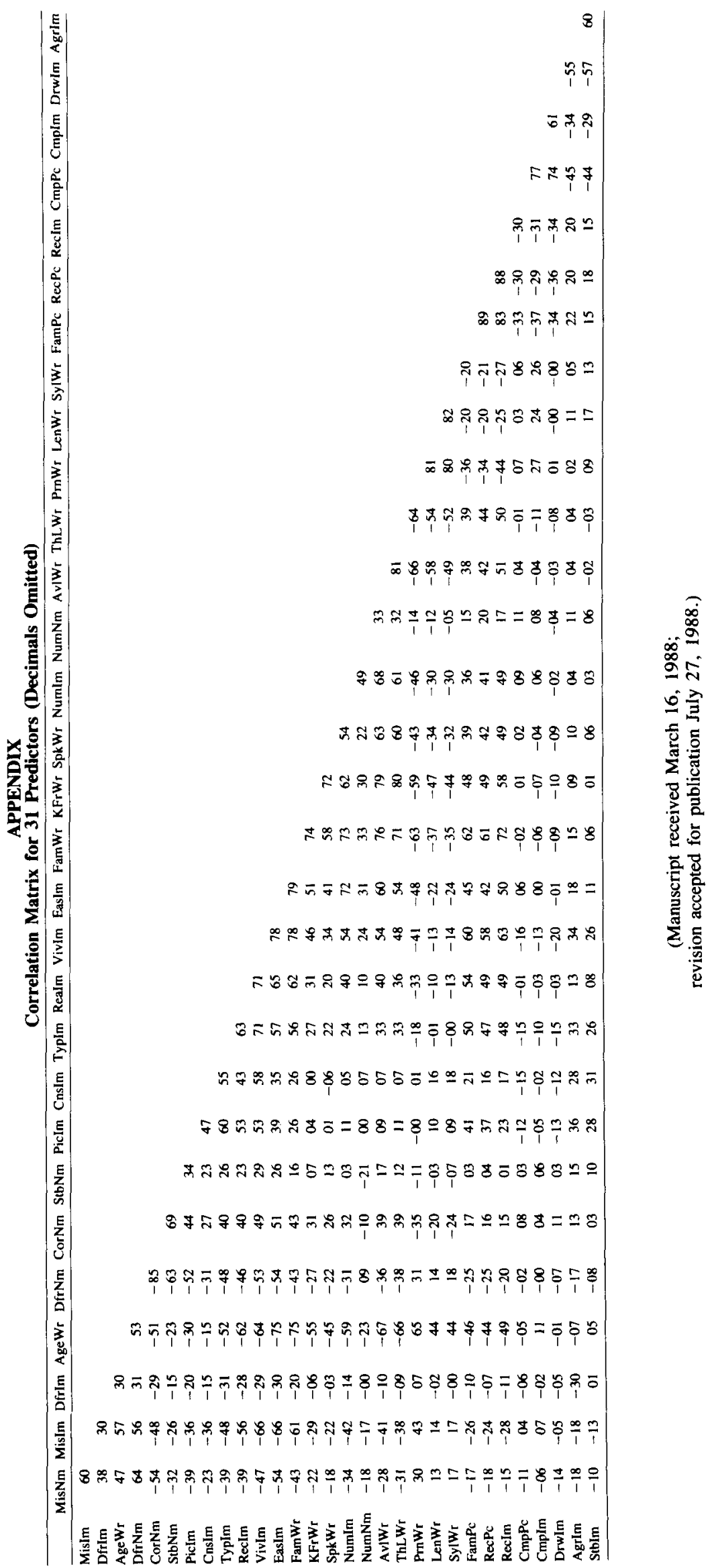

